\title{
On macroscopic dimension of rationally essential manifolds
}

\author{
AlEXANDER DRANishniKov
}

\begin{abstract}
We construct a counterexamples in dimensions $n>3$ to Gromov's conjecture [11] that the macroscopic dimension of rationally essential $n$-dimensional manifolds equals $n$.
\end{abstract}

53C23; 20J06, 55N91, 55M10, 57N65

\section{Introduction}

Gromov brought to life several definitions of a large scale dimension. Perhaps the best known of these is the notion of the asymptotic dimension introduced as an invariant of discrete groups [10]. It turned out that the finiteness of asymptotic dimension for a group implies many famous conjectures of Novikov's type for that group. The asymptotic dimension asdim $X$ is defined for general metric spaces $X$ and by its nature does not take into account the local structure of a space. The notion of macroscopic dimension $\operatorname{dim}_{\mathrm{mc}} X$ was introduced by Gromov for studying Riemannian manifolds [11]. This notion of large-scale dimension is sensitive to the local structure in particular, to the covering dimension of a space which by definition is local. We note that always $\operatorname{dim}_{\mathrm{mc}} X \leq \operatorname{asdim} X$.

Gromov stated several conjectures and questions concerning macroscopic dimension. One of his conjectures on $\operatorname{dim}_{\mathrm{mc}}$ was that the universal covering $\widetilde{M}$ of any $n$-manifold $M$ with positive scalar curvature satisfies the inequality $\operatorname{dim}_{\mathrm{mc}} \widetilde{M} \leq n-2$. This conjecture seems to be out of reach since it implies the Gromov-Lawson conjecture about non-existence of a positive scalar curvature metric on any closed aspherical manifold. The latter is known to be a Novikov type conjecture. We refer to the paper by Bolotov and the author [3] for recent progress on the Gromov scalar curvature conjecture modulo the Novikov conjecture. In this paper we solve another Gromov's problem which connects the macroscopic dimension of the universal covering with the essentiality of the manifold.

Gromov studied the question when the universal covering $\widetilde{M}$ of a closed $n$-dimensional manifold could have macroscopic dimension strictly less than $n$. The main source of $n$-manifolds satisfying the inequality $\operatorname{dim}_{\mathrm{mc}} \widetilde{M}<n$ is the class of inessential manifolds 
also introduced by Gromov [9]. We recall that an $n$-manifold $M$ is called inessential if a map $f: M \rightarrow B \pi$ that classifies its universal covering $\widetilde{M}$ can be deformed to the $(n-1)$-skeleton $B \pi^{(n-1)}$. Otherwise it is called essential. It is well-known that an orientable manifold is inessential if and only if the image of its fundamental class under the induced homomorphism $f_{*}: H_{*}(M) \rightarrow H_{*}(B \pi)$ is zero, $f_{*}([M])=0$ (see [3]). An example of an essential $n$-manifold $M$ with $\operatorname{dim}_{\text {mc }} \widetilde{M}<n$ is the real projective space $\mathbb{R} P^{n}$. Though $M=\mathbb{R} P^{n}$ is essential it is rationally inessential for odd $n$, that is, with $f_{*}([M])=0$ in $H_{*}(B \pi ; \mathbb{Q})$. Gromov conjectured this is always the case.

Conjecture 1.1 (Gromov [11], $2 \frac{2}{3}$ Remarks and Questions) If $\operatorname{dim}_{\mathrm{mc}} \widetilde{M}<n$ where $\widetilde{M}$ is the universal covering of a closed orientable $n$-manifold $M$, then $M$ must be rationally inessential.

The main goal of this paper to give counterexamples to Gromov's conjecture. For that we define a new notion of macroscopic dimension $\operatorname{dim}_{M C}$ satisfying the inequality

$$
\operatorname{dim}_{\mathrm{mc}} X \leq \operatorname{dim}_{\mathrm{MC}} X \leq \operatorname{asdim} X
$$

and construct rationally essential closed $n$-manifolds $M$ with $\operatorname{dim}_{\mathrm{MC}} \widetilde{M}<n$.

Definition 1.2 (Gromov [11]) A metric space $X$ has the macroscopic dimension less or equal to $k, \operatorname{dim}_{\mathrm{mc}} X \leq k$, if there is a continuous uniformly cobounded map $f: X \rightarrow N^{k}$ to a $k$-dimensional simplicial complex.

A map $f: X \rightarrow Y$ of a metric space is uniformly cobounded if there is a constant $C>0$ such that $\operatorname{diam}\left(f^{-1}(y)\right)<C$ for all $y \in Y$.

Definition 1.3 A metric space $X$ has the macroscopic dimension $\operatorname{dim}_{\mathrm{MC}} X \leq k$, if there is a Lipschitz uniformly cobounded map $f: X \rightarrow N^{k}$ to a $k$-dimensional simplicial complex.

Here we assume that a simplicial complex $N$ has a metric inherited from the Hilbert space $\ell_{2}\left(N^{(0)}\right)$ spanned by the vertices of $N$ under the canonical embedding into the standard simplex $N \subset \Delta \subset \ell_{2}\left(N^{(0)}\right)$. We will call $\operatorname{dim}_{\mathrm{MC}}$ the macroscopic dimension and will refer to $\operatorname{dim}_{\mathrm{mc}}$ as Gromov's macroscopic dimension. Note that $\operatorname{dim}_{\mathrm{mc}}$ and $\operatorname{dim}_{\mathrm{MC}}$ are invariant of quasi-isometry homeomorphisms. Therefore, the macroscopic dimensions $\operatorname{dim}_{\mathrm{mc}} \widetilde{M}$ and $\operatorname{dim}_{\mathrm{MC}} \widetilde{M}$ of the universal covering $\widetilde{M}$ of a closed manifold $M$ taken with the lifted from $M$ metric do not depend on the choice of the metric on $M$.

Clearly,

$$
\operatorname{dim}_{\mathrm{mc}} X \leq \operatorname{dim}_{\mathrm{MC}} X .
$$


The original definition of asymptotic dimension uses coverings by large open sets. Alternatively, the asymptotic dimension can be defined as follows (see Gromov [10], and the paper by Bell and the author [1]):

Definition 1.4 A metric space $X$ has asymptotic dimension less than or equal to $k$, asdim $X \leq k$, if for every $\epsilon>0$ there is an $\epsilon$-Lipschitz uniformly cobounded map $f: X \rightarrow N^{k}$ to a $k$-dimensional simplicial complex.

Then, clearly,

$$
\operatorname{dim}_{\mathrm{MC}} X \leq \operatorname{asdim} X .
$$

In this paper we develop a cohomological approach to macroscopic dimension outlined by the author in [6]. This theory combined with a homological characterization of amenability given by Block and Weinberger [2] produces the following examples.

Theorem 1.5 For all $n \geq 4$ there are closed rationally essential $n$-manifolds $M$ with $\operatorname{dim}_{\mathrm{MC}} \widetilde{M}<n$.

The non-amenability of the fundamental group in these examples is essential in view of the following

Theorem 1.6 (Dranishnikov [6]) For rationally essential $n$-manifolds with amenable fundamental group there is the equality $\operatorname{dim}_{\mathrm{MC}} \widetilde{M}=n$.

It turns out that the inequality $\operatorname{dim}_{\mathrm{MC}} \widetilde{M}<n$ depends only on the homology class $\alpha=f_{*}([M]) \in H_{n}(B \pi)$ where $f: M \rightarrow B \pi$ is a map that classifies the universal covering of $M$. It means that for any manifold $N$ with classifying map $g: N \rightarrow B \pi$ and $g_{*}([N])=\alpha$ it follows that $\operatorname{dim}_{\mathrm{MC}} \tilde{N}<n$. Following Brunnbauer and Hanke [5] we call such classes small. It turns out that small classes form a subgroup in $H_{n}(B \pi)$. This phenomenon was discovered first in [5] with respect to many classes of so-called large manifolds such as manifolds with hyper-spherical universal cover, with hyper-euclidean universal cover, enlargeable, etc. The property $\operatorname{dim}_{\mathrm{MC}} \widetilde{M}=\operatorname{dim} M$ for a manifold $M$ certainly represents some largeness. We call such manifolds $M$ macroscopic dimension large. Nevertheless Brunnbauer-Hanke approach does not work here. In particular, for all largeness conditions treated in [5] the torsion elements are small. It is still an open question whether torsion elements of $H_{*}(B \pi)$ are small for the macroscopic dimension large property. Our proof that small classes in $H_{*}(B \pi)$ for the macroscopic dimension largeness form a subgroup is based on a concept of the almost equivariant 
(co)homology introduced in this paper. Curiously, the work on that proof brought as the byproduct the following formula for homology of groups:

$$
H_{n}(B \pi)=\operatorname{ker}\left\{I^{n} \otimes_{\pi} \mathbb{Z} \stackrel{(1 \otimes j) \otimes 1}{\longrightarrow}\left(I^{n-1} \otimes \mathbb{Z} \pi\right) \otimes_{\pi} \mathbb{Z}\right\}
$$

where $j: I \rightarrow \mathbb{Z} \pi$ is the inclusion of the augmentation ideal $I$ into the group ring $\mathbb{Z} \pi$ and $I^{n}=I \otimes \cdots \otimes I$ is the $n$th tensor power over $\mathbb{Z}$.

\section{Almost equivariant cohomology}

Let $X$ be a CW complex and let $E_{n}(X)$ denote the set of its $n$-dimensional cells. We recall that (co)homology of a CW complex $X$ with coefficients in an abelian group $G$ are defined by by means of the cellular chain complex $C_{*}(X)=\left\{C_{n}(X), \partial_{n}\right\}$ where $C_{n}(X)$ is the free abelian group generated by the set $E_{n}(X)$. The resulting groups $H_{*}(X ; G)$ and $H^{*}(X ; G)$ do not depend on the choice of the CW structure on $X$. The proof of this fact appeals to the singular (co)homology theory and it is a part of all textbooks on algebraic topology. The same holds true for (co)homology groups with locally finite coefficients, that is, for coefficients in a $\pi$-module $L$ where $\pi=\pi_{1}(X)$. The chain complex defining the homology groups $H_{*}(X ; L)$ is $\left\{C_{n}(\tilde{X}) \otimes_{\pi} L\right\}$ and the cochain complex defining the cohomology $H^{*}(X ; L)$ is $\operatorname{Hom}_{\pi}\left(C_{n}(\tilde{X}), L\right)$ where $\tilde{X}$ is the universal cover of $X$ with the cellular structure induced from $X$. The resulting groups $H_{*}(X ; L)$ and $H^{*}(X ; L)$ do not depends on the CW structure on $X$.

These groups can be interpreted as the equivariant (co)homology:

$$
H_{*}(X ; L)=H_{*}^{l f, \pi}(\tilde{X} ; L) \text { and } H^{*}(X ; L)=H_{\pi}^{*}(\tilde{X} ; L) .
$$

The last equality is obvious since the equivariant cohomology groups $H_{\pi}^{*}(\tilde{X} ; L)$ are defined by equivariant cochains $\operatorname{Hom}_{\pi}\left(C_{n}(\tilde{X}), L\right\}$. We recall that the equivariant locally finite homology groups are defined by the complex of infinite locally finite invariant chains

$$
C_{n}^{l f, \pi}(\tilde{X} ; L)=\left\{\sum_{e \in E_{n}(\tilde{X})} \lambda_{e} e \mid \lambda_{g e}=g \lambda_{e}, \lambda_{e} \in L\right\} .
$$

The local finiteness condition on a chain requires that for every $x \in \tilde{X}$ there is a neighborhood such that the number of $n$-cells $e$ intersecting $U$ for which $\lambda_{e} \neq 0$ is finite. This condition is satisfied automatically when $X$ is a locally finite complex. Even in that case if is the part the notation for the equivariant homology since it was inherited from the singular theory. The following Proposition implies the equality $H_{*}(X ; L)=H_{*}^{l f, \pi}(\tilde{X} ; L)$. 
Proposition 2.1 For every $C W$ complex $X$ with the fundamental group $\pi$ and a $\pi$-module $L$ the chain complex $\left\{C_{n}(\tilde{X}) \otimes_{\pi} L\right\}$ is isomorphic to the chain complex of locally finite equivariant chains $C_{*}^{\pi}(\tilde{X})$.

The following definition was given in the author's paper [6] in more general setting.

Definition 2.2 Let $X$ be a CW complex with the universal cover $\tilde{X}$ and let $L$ be a $\pi$-module. A homomorphism $\phi: C_{n}(\tilde{X}) \rightarrow L$ is called almost equivariant, if the set

$$
\left\{\gamma^{-1} \phi(\gamma e) \mid \gamma \in \pi\right\} \subset L
$$

is finite for every $n$-cell $e$ in $\tilde{X}$. Let $\operatorname{Hom}_{a e}\left(C_{n}(\tilde{X}), L\right)$ be the set of all almost equivariant homomorphisms from $C_{n}(\tilde{X})$ to $L$. Note that this is a group. These groups form a cochain complex with respect to the co-differential $\delta$ defined as $(\delta f)(\Delta)=f(\partial \Delta)$ where $\partial: C_{n}(\tilde{X}) \rightarrow C_{n-1}(\tilde{X})$ are the boundary homomorphisms. The cohomology groups $H_{a e}^{*}(\tilde{X} ; L)$ are called the almost equivariant cohomology of $\tilde{X}$ with coefficients in a $\pi$-module $L$.

One can define singular almost equivariant cohomology by replacing $n$-cells $e$ in the above definition by singular simplices $\sigma: \Delta^{n} \rightarrow \tilde{X}$. The standard argument show that the singular version of almost equivariant cohomology coincides with the cellular. Thus the group $H_{a e}^{*}(\tilde{X} ; L)$ does not depend on the choice of a CW complex structure on $X$. Clearly, for complexes $X$ with finite fundamental group there is an isomorphism $H_{a e}^{*}(\tilde{X} ; L)=H^{*}(\tilde{X} ; L)$.

Since every equivariant homomorphism is almost equivariant, there is a natural transformation

$$
\operatorname{pert}_{X}^{*}: H^{*}(X ; L)=H_{\pi}^{*}(\tilde{X} ; L) \rightarrow H_{a e}^{*}(\tilde{X} ; L)
$$

called a perturbation homomorphism from the cohomology of $X$ to the almost equivariant cohomology.

We note that in the case when $L=\mathbb{Z}$, a trivial $\pi$-module, the groups $H_{a e}^{*}(\tilde{X} ; \mathbb{Z})$ coincide with the $\ell_{\infty}$-cohomology groups $H_{(\infty)}^{*}(\tilde{X} ; \mathbb{Z})$ defined by Gersten [8].

Also we note that a proper cellular map $f: X \rightarrow Y$ that induces an isomorphism of the fundamental groups lifts to a proper cellular map of the universal covering spaces $\bar{f}: \tilde{X} \rightarrow \tilde{Y}$. The lifting $\bar{f}$ defines a chain homomorphism $\bar{f}_{*}: C_{n}(\tilde{X}) \rightarrow C_{n}(\tilde{Y})$ and a cochain homomorphism $\bar{f}^{*}: \operatorname{Hom}_{a e}\left(C_{n}(\tilde{Y}), L\right) \rightarrow \operatorname{Hom}_{a e}\left(C_{n}(\tilde{X}), L\right)$. The latter defines a homomorphisms of the almost equivariant cohomology groups

$$
\bar{f}_{a e}^{*}: H_{a e}^{*}(\tilde{Y} ; L) \rightarrow H_{a e}^{*}(\tilde{X} ; L) .
$$


Suppose that $\pi$ acts freely on CW complexes $\tilde{X}$ and $\tilde{Y}$ such that the actions preserve the CW complex structures. We call a cellular map $g: \tilde{X} \rightarrow \tilde{Y}$ almost equivariant if the set

$$
\bigcup_{\gamma \in \pi}\left\{\gamma^{-1} g_{*}(\gamma e)\right\} \subset C_{*}(\tilde{Y})
$$

is finite for every cell $e$ in $\tilde{X}$ where $g_{*}: C_{*}(\tilde{X}) \rightarrow C_{*}(\tilde{Y})$ the induced chain map.

Proposition 2.3 Let $f: X \rightarrow Y$ be a proper almost equivariant cellular map. Then the induced homomorphism on cochains takes the almost equivariant cochains to almost equivariant.

Proof Let $\phi: C_{n}(Y) \rightarrow L$ be an almost equivariant cochain. Let $e^{\prime}$ be an $n$-cell in $X$. There are finitely many chains $c_{1}, \ldots, c_{m} \in C_{n}(\tilde{Y})$ such that $f\left(\gamma e^{\prime}\right) \subset \gamma\left\{c_{1}, \ldots, c_{m}\right\}$ for all $\gamma \in \pi$. Then the set

$$
\bigcup_{\gamma \in \pi}\left\{\gamma^{-1} \phi\left(f\left(\gamma e^{\prime}\right)\right)\right\} \subset \bigcup_{\gamma \in \pi}\left\{\gamma^{-1} \phi\left(\gamma\left\{c_{1}, \ldots, c_{m}\right\}\right)\right\}=\bigcup_{i=1}^{m} \bigcup_{\gamma \in \pi}\left\{\gamma^{-1} \phi\left(\gamma c_{i}\right)\right\}
$$

is finite.

For every CW-complex $X$ we consider the product CW-complex structure on $X \times[0,1]$ with the standard cellular structure on $[0,1]$.

Proposition 2.3 and the standard facts about cellular chain complexes imply the following.

Proposition 2.4 Let $X$ and $Y$ be complexes with free cellular actions of a group $\pi$.

(A) Then every almost equivariant cellular map $f: X \rightarrow Y$ induces an homomorphism of the almost equivariant cohomology groups

$$
f^{*}: H_{a e}^{*}(Y ; L) \rightarrow H_{a e}^{*}(X ; L) .
$$

(B) If two almost equivariant maps $f_{1}, f_{2}: X \rightarrow Y$ are homotopic by means of a cellular almost equivariant homotopy $H: X \times[0,1] \rightarrow Y$, then they induce the same homomorphism of the almost equivariant cohomology groups, $f_{1}^{*}=f_{2}^{*}$.

Similarly one can define the almost equivariant homology groups on a CW complex by considering infinite locally finite almost equivariant chains. Let $X$ be a complex with the fundamental group $\pi$ and the universal cover $\tilde{X}$. We call an infinite chain $\sum_{e \in E_{n}(\tilde{X})} \lambda_{e} e$ almost equivariant if the set $\left\{\gamma^{-1} \lambda_{\gamma e} \mid \gamma \in \pi\right\} \subset L$ is finite for every cell $e$. As we already have mentioned, the complex of equivariant locally finite 
chains defines equivariant locally finite homology $H_{*}^{l f, \pi}(\tilde{X} ; L)$. The homology defined by the almost equivariant locally finite chain we call the almost equivariant locally finite homology. We denote them as $H_{*}^{l f, a e}(\tilde{X} ; L)$. We note that like in the case of cohomology this definition can be carried out for the singular homology and it gives the same groups. In particular the groups $H_{*}^{l f, a e}(\tilde{X} ; L)$ do not depend on the choice of a CW complex structure on $X$.

We note that when $L=\mathbb{Z}$ is a trivial $\pi$-module, the almost equivariant locally finite homology groups $H_{*}^{l f}$,ae $(\tilde{K} ; L)$ coincide with the uniformly finite homology $H_{*}^{u f}(\tilde{K} ; \mathbb{Z})$ defined by Block and Weinberger [2].

As in the case of cohomology for any complex $K$ there is a perturbation homomorphism

$$
\operatorname{pert}_{*}^{K}: H_{*}(K ; L)=H_{*}^{l f, \pi}(\tilde{K} ; L) \rightarrow H_{*}^{l f, a e}(\tilde{K} ; L) .
$$

Also, there is an analog of Proposition 2.4 for the almost equivariant locally finite homology.

Proposition 2.5 Let $X$ and $Y$ be complexes with free cellular actions of a group $\pi$.

(A) Then every almost equivariant cellular map $f: X \rightarrow Y$ induces a homomorphism of the almost equivariant homology groups

$$
f_{*}: H_{*}^{l f, a e}(X ; L) \rightarrow H_{*}^{l f, a e}(Y ; L) .
$$

(B) If two almost equivariant maps $f_{1}, f_{2}: X \rightarrow Y$ are homotopic by means of a cellular almost equivariant homotopy, then they induce the same homomorphism of the almost equivariant cohomology groups, $\left(f_{1}\right)_{*}=\left(f_{2}\right)_{*}$.

Let $X_{i}, i=1,2$ be complexes with free action of $\pi_{i}$ and $L_{i}$ be $\pi_{i}$-modules. The tensor product on locally finite chains

$$
C_{k}^{l f}\left(X_{1}, L_{1}\right) \otimes C_{l}^{l f}\left(X_{2}, L_{2}\right) \rightarrow C_{k+l}^{l f}\left(X_{1} \times X_{2}, L_{1} \otimes L_{2}\right)
$$

defined by the formula

$$
\sum m_{\sigma} \sigma \otimes \sum n_{\kappa} \kappa \rightarrow \sum\left(m_{\sigma} \otimes n_{\kappa}\right)(\sigma \times \kappa)
$$

takes the product of almost equivariant chains to almost equivariant. Since the above tensor product defines homomorphisms $\phi_{*}$ and $\phi_{*}^{a e}$ for both the equivariant and the almost equivariant homology, we obtain the following: 
Proposition 2.6 For any complexes $M$ and $N$ with universal coverings $\widetilde{M}$ and $\tilde{N}$, for any $\pi_{1}(M)$ and $\pi_{1}(N)$ modules $L_{1}$ and $L_{2}$, and for any $k$ and $l$, there is a commutative diagram:

$$
\begin{array}{ccc}
H_{k}\left(M ; L_{1}\right) \otimes H_{l}\left(N ; L_{2}\right) & \stackrel{\phi_{*}}{\longrightarrow} H_{k+l}\left(M \times N ; L_{1} \otimes L_{2}\right) \\
\operatorname{pert}_{*}^{M} \otimes \operatorname{pert}_{*}^{N} \downarrow & & \operatorname{pert}_{*}^{M \times N} \downarrow \\
H_{k}^{l f, a e}\left(\widetilde{M} ; L_{1}\right) \otimes H_{l}^{l f, a e}\left(\tilde{N} ; L_{2}\right) \stackrel{\phi_{*}^{a e}}{\longrightarrow} H_{k+l}^{l f \cdot a e}\left(\widetilde{M} \times \tilde{N} ; L_{1} \otimes L_{2}\right) .
\end{array}
$$

Let $M$ be an oriented $n$-dimensional PL manifold with a fixed triangulation. Denote by $M^{*}$ the dual complex. There is a bijection between $k$-simplices $e$ and the dual $(n-k)-$ cells $e^{*}$ which defines the Poincare duality isomorphism. This bijection extends to a similar bijection on the universal cover $\widetilde{M}$. Let $\pi=\pi_{1}(M)$. For any $\pi$-module $L$ the Poincare duality on $M$ with coefficients in $L$ is given by the cochain-chain level isomorphisms

$$
\operatorname{Hom}_{\pi}\left(C_{k}\left(\widetilde{M}^{*}\right), L\right) \stackrel{P D_{k}}{\longrightarrow} C_{n-k}^{l f, \pi}(\widetilde{M} ; L)
$$

where $P D_{k}$ takes a cochain $\phi: C_{k}\left(\widetilde{M}^{*}\right) \rightarrow L$ to the chain $\sum_{e \in E_{n-k}(\widetilde{M})} \phi\left(e^{*}\right) e$. The family $P D_{*}$ is a chain isomorphism which is also known as the cap product

$$
P D_{k}(\phi)=\phi \cap[\widetilde{M}]
$$

with the fundamental class $[\widetilde{M}] \in C_{n}^{l f, \pi}(\widetilde{M})$, where $[\widetilde{M}]=\sum_{e \in E_{n}(\widetilde{M})} e$. We note that the homomorphisms $P D_{k}$ and $P D_{k}^{-1}$ extend to the almost equivariant chains and cochains:

$$
\operatorname{Hom}_{a e}\left(C_{k}\left(\widetilde{M}^{*}\right), L\right) \stackrel{P D_{k}}{\longrightarrow} C_{n-k}^{l f, a e}(\widetilde{M} ; L) .
$$

Thus, the homomorphisms $P D_{*}$ define the Poincare duality isomorphisms $P D_{a e}$ between the almost equivariant cohomology and homology. We summarize this in the following

Proposition 2.7 For any closed oriented $n$-manifold $M$ and any $\pi_{1}(M)$-module $L$ the Poincare duality forms the following commutative diagram:

$$
\begin{array}{ccc}
H^{k}(M ; L) & \stackrel{\operatorname{pert}_{M}^{*}}{\longrightarrow} & H_{a e}^{k}(\widetilde{M} ; L) \\
-\cap[M] \downarrow & P D_{a e} \downarrow \\
H_{n-k}(M ; L) \stackrel{\operatorname{pert}_{*}^{M}}{\longrightarrow} & H_{n-k}^{l f, a e}(\widetilde{M} ; L) .
\end{array}
$$


We note that the operation of the cap product for equivariant homology cohomology automatically extends on the chain-cochain level to the cap product on the almost equivariant homology and cohomology. Then the Poincare Duality isomorphism $P D_{a e}$ for $\widetilde{M}$ can be described as the cap product with the homology class $\operatorname{pert}_{*}^{M}([M])$.

\section{Obstruction to the inequality $\operatorname{dim}_{\mathrm{MC}} M^{n}<n$}

Let $\pi$ be a finitely presented group. Then the classifying space $B \pi=K(\pi, 1)$ can be taken to be a locally finite complex. We fix a geodesic metric on $B \pi$. Let $p_{\pi}: E \pi \rightarrow$ $B \pi$ denote the universal covering. We consider the induced $\mathrm{CW}$ complex structure and induced geodesic metric on $E \pi$.

Proposition 3.1 Let $K$ be a finite complex with the universal cover $p: \widetilde{K} \rightarrow K$ supplied by a geodesic metric induced from $K$. Let $f: K \rightarrow B \pi$ be a cellular Lipschitz map classifying $p$. Suppose that $\operatorname{dim}_{\mathrm{MC}} \tilde{K}<n$. Then for every lift $\tilde{f}: \widetilde{K} \rightarrow E \pi$ of $f p$ there is a Lipschitz cellular homotopy $H: \widetilde{K} \times I \rightarrow E \pi$ of $\tilde{f}$ to a map $g: \widetilde{K} \rightarrow E \pi^{(n-1)}$ where $E \pi^{(n-1)}$ denotes the $(n-1)$-skeleton of $E \pi$.

Proof We may assume that $B \pi$ is a locally finite complex. Moreover, since the group $\pi=\pi_{1}(K)$ is finitely presented, we may assume that the $2-$ skeleton $B \pi^{(2)}$ is a finite complex. Let $\phi: \widetilde{K} \rightarrow N$ be a uniformly cobounded Lipschitz map to an $(n-1)-$ dimensional simplicial complex. We may assume that $\phi$ is cellular and surjective on the cell level, that is an every cell in $N$ has nonempty intersection with the image $\phi(\tilde{K})$. Also we may assume that there is $C>0$ such that $\operatorname{diam}\left(\phi^{-1}(\Delta)\right)<C$ of all simplices $\Delta$ in $N$. We construct a Lipschitz map $q: N \rightarrow E \pi^{(n-1)}$ by induction on dimension of the skeleton of $N$. For every $v \in N^{(0)}$ we define $q(v)$ be a closest vertex in $E \pi$ to the set $\tilde{f}\left(\phi^{-1}(v)\right)$. Then for every edge $\left[v, v^{\prime}\right]$ in $N$ we define $q\left(\left[v, v^{\prime}\right]\right)$ to be a shortest path in the Cayley graph $E \pi^{(1)}$ taken with the graph metric from $q(v)$ to $q\left(v^{\prime}\right)$. Since the distance between $q(v)$ and $q\left(v^{\prime}\right)$ for all edges $\left[v, v^{\prime}\right]$ is uniformly bounded, there is an upper bound on the number of isometry types of such paths. Then for every 2-simplex $\sigma=\left[v_{0}, v_{1}, v_{2}\right]$ is $N$ we take a filing of $q(\partial \sigma)$ in $E \pi^{(2)}$ that uses a minimal number of $2-$ cells and so on.

Let $\mathcal{H}_{k}$ denote the set of all simplicial embeddings $h: \Delta^{k} \rightarrow N$ of the standard $k-$ simplex. After the step number $k<n$ we will get a Lipschitz map $q: N^{(k)} \rightarrow E \pi^{(k)}$ such that the family of maps $\left\{p_{\pi} q h: \partial \Delta^{k+1} \rightarrow B \pi\right\}_{h \in \mathcal{H}_{k}}$ is finite. Then we can construct a Lipschitz extension $q: N^{(k+1)} \rightarrow E \pi^{(k+1)}$ using minimal fillings in $B \pi$. As the result we obtain a Lipschitz map $q: N \rightarrow E \pi^{(n)}$ with $p_{\pi} q(N)$ compact. We note that the composition $q \phi$ is on bounded distance from $\widetilde{f}$. Clearly, the maps 
$\tilde{f}$ and $q \phi$ are homotopic as maps to a contractible space. Since $q \phi$ and $\tilde{f}$ are on bounded distance with compact projections $p_{\pi} \tilde{f}(\tilde{X})$ and $p_{\pi} q \phi(\tilde{X})$, there is a uniformly bounded homotopy between them. Then we can turn that homotopy to a cellular Lipschitz map.

Let $A$ be a subset of a CW complex $X$. The star neighborhood $\operatorname{St}(A)$ of $A$ is the closure of the union of all cells in $X$ that have a nonempty intersection with $A$. Note that $\operatorname{St}(A)$ is a subcomplex of $X$.

Proposition 3.2 Let $X$ and $Y$ be as above with $Y$ locally finite. Then a cellular Lipschitz homotopy $\Phi: X \times[0,1] \rightarrow Y$ of an almost equivariant map is almost equivariant.

Proof Since $\left.\Phi\right|_{X \times\{0\}}$ is almost equivariant, for every cell $e \subset X$ the union

$$
\bigcup_{\gamma \in \pi} \Phi(e \times\{0\}) \subset \sigma_{1} \cup \cdots \cup \sigma_{k} .
$$

Then

$$
\bigcup_{\gamma \in \pi} \Phi(e \times(0,1)) \subset \mathrm{St}^{m}(K)
$$

where $K$ is the closure of $\sigma_{1} \cup \cdots \cup \sigma_{k}$. The existence of $m$ follows from the fact that $\Phi$ is Lipschitz. The local finiteness of $Y$ and finiteness of $K$ imply that the $m$-times iterated star neighborhood $\mathrm{St}^{m}(K)$ of $K$ is a finite subcomplex of $Y$. Clearly, the coefficients of the cells in $\Phi_{*}(e \times(0,1)) \in C_{*}(Y)$ are bounded.

Here we recall some basic facts of the elementary obstruction theory. Let $f: X \rightarrow Y$ be a cellular map that induces an isomorphism of the fundamental groups. We want to deform the map $f$ to a map to the $(n-1)$-skeleton $Y^{(n-1)}$. For that we consider the extension problem

$$
X \supset X^{(n-1)} \stackrel{f}{\rightarrow} Y^{(n-1)},
$$

that is, the problem to extend $f: X^{(n-1)} \rightarrow Y^{(n-1)}$ continuously to a map $\bar{f}: X \rightarrow$ $Y^{(n-1)}$. The primary obstruction for this problem $o_{f}$ is the obstruction to extend $f$ to the $n$-skeleton. It lies in the cohomology group $H^{n}(X ; L)$ where $L=\pi_{n-1}\left(Y^{(n-1)}\right)$ is the $(n-1)$-dimensional homotopy group considered as a $\pi$-module for $\pi=\pi_{1}(Y)=$ $\pi_{1}(X)$. The obstruction theory says that a map $g: X \rightarrow Y^{(n-1)}$ that agrees with $f$ on the (n-2)-skeleton $X^{(n-2)}$ exits if and only if $o_{f}=0$. The primary obstruction is natural: If $g: Z \rightarrow X$ is a cellular map, then $o_{g f}=g^{*}\left(o_{f}\right)$. In particular, in our case $o_{f}=f^{*}\left(o_{1}\right)$ where $o_{1} \in H^{n}(Y ; L)$ is the primary obstruction to the retraction of $Y$ to the $(n-1)$-skeleton. 
Definition 3.3 Let $g: Y^{(n-1)} \rightarrow Z$ be a Lipschitz map of the $(n-1)$-skeleton of an $n$-dimensional complex to a metric space. We call the problem to extend $g$ to a Lipschitz map $\bar{g}: Y \rightarrow Z$ a Lipschitz extension problem.

Definition 3.4 Let $X$ be a finite $n$-complex, $n \geq 3$, with $\pi_{1}(X)=\pi$ and $\tilde{f}: \tilde{X} \rightarrow \tilde{Y}$ be a lift of a cellular map $f: X \rightarrow Y$ that induces an isomorphism of the fundamental groups. We define an element $o \tilde{f} \in H_{a e}^{n}\left(\tilde{X} ; \pi_{n-1}\left(Y^{(n-1)}\right)\right)$ as the class of the cocycle

$$
C_{\tilde{f}}: C_{n}(\tilde{X}) \rightarrow \pi_{n-1}\left(\tilde{Y}^{(n-1)}\right)=\pi_{n-1}\left(Y^{(n-1)}\right)
$$

defined by the formula $C_{\tilde{f}}(e)=\left[\tilde{f} \circ \phi_{e}\right]$ where $\phi_{e}: S^{n-1}=\partial B^{n} \rightarrow X$ is the attaching map of an $n$-cell $e$. Since $\tilde{f}$ is $\pi$-equivariant, the cocycle $C_{\tilde{f}}$ is a $\pi$-equivariant. Thus, it defines an element $\kappa_{f} \in H_{\pi}^{n}\left(\tilde{X} ; \pi_{n-1}\left(Y^{(n-1)}\right)\right)$ of the equivariant cohomology and $o_{\tilde{f}}=\operatorname{pert}_{X}^{*}\left(\kappa_{f}\right)$.

We consider an arbitrary geodesic metric on a locally finite complex and the induced metric on its universal cover.

Proposition 3.5 Let $\tilde{f}: \tilde{X} \rightarrow \tilde{Y}$ be a lift of a Lipschitz cellular map $f: X \rightarrow Y$ of a finite $n$-dimensional complex to a locally finite that induces an isomorphism of the fundamental groups. Then the above cohomology class $o \tilde{f} \in H_{a e}^{n}\left(\tilde{X} ; \pi_{n-1}\left(Y^{(n-1)}\right)\right)$ is the primary obstruction for the following Lipschitz extension problem

$$
\tilde{X} \supset \tilde{X}^{(n-1)} \stackrel{\tilde{f} \mid}{\rightarrow} \tilde{Y}^{(n-1)} .
$$

Thus, $o_{\tilde{f}}=0$ if and only if there is a Lipschitz map $\bar{g}: \tilde{X} \rightarrow \tilde{Y}^{(n-1)}$ which agrees with $\widetilde{f}$ onto $\widetilde{X}^{(n-2)}$.

Proof The proof goes along the lines of a similar statement from the classical obstruction theory. Let $C_{\tilde{f}}=\delta \Psi$ where $\Psi: C_{n-1}(\tilde{X}) \rightarrow \pi_{n-1}\left(\tilde{Y}^{(n-1)}\right)$ be an almost equivariant homomorphism. For each $(n-1)-$ cell $e$ of $X$ we fix a section $\tilde{e} \subset \tilde{X}$, an $(n-1)$-cell in $\tilde{X}$. Then the set $\left\{\gamma^{-1} \Psi(\gamma \widetilde{e}) \mid \gamma \in \pi\right\}=\left\{\gamma_{i}^{-1} \Psi\left(\gamma_{i} \widetilde{e}\right)\right\}_{i=1}^{m}$ is finite. Like in the classical obstruction theory we define a map $g_{i}: \gamma_{i} \tilde{e} \rightarrow Y^{(n-1)}, i=1, \ldots, m$ on cells $\gamma_{i} \tilde{e}$ such that $g_{i}$ agrees with $\widetilde{f}$ outside a small $(n-1)-$ ball $B_{i} \subset \gamma_{i} \tilde{e}$. The difference of $\widetilde{f}$ and $g_{i}$ restricted to $B_{i}$ defines a map $d_{\tilde{f}, g_{i}}: S^{n-1} \rightarrow Y^{(n-1)}$ that represents the class $-\Psi\left(\gamma_{i} \widetilde{e}\right)$. For a general lift $\gamma \widetilde{e}$ of $e$ we define a map $g: \gamma \widetilde{e} \rightarrow Y^{(n-1)}$ as follows. Let $i$ be such that $\gamma^{-1} \Psi(\gamma \widetilde{e})=\gamma_{i}^{-1} \Psi\left(\gamma_{i} \widetilde{e}\right)$. We define $g=\gamma \gamma_{i}^{-1} g_{i} \gamma_{i} \gamma^{-1}$. Thus, we define $g: \tilde{X}^{(n-1)} \rightarrow \tilde{Y}^{(n-1)}$ in such a way that the difference map $d_{\tilde{f}, g}: S^{n-1} \rightarrow Y^{(n-1)}$ on the cell $\gamma \tilde{e}, \gamma \in \pi$, represents the element $-\left(\gamma_{i} \gamma\right)^{-1} \Psi\left(\gamma_{i} \widetilde{e}\right) \stackrel{\tilde{X}, g}{=}-\Psi(\gamma \widetilde{e})$. Then the elementary obstruction theory implies that for every $n-$ cell $\sigma^{\prime} \subset \tilde{X}$ there is an extension 
$\bar{g}_{\sigma^{\prime}}: \overline{\sigma^{\prime}} \rightarrow \tilde{Y}^{(n-1)}$ of $\left.g\right|_{\partial \sigma^{\prime}}$. For every $n$-cell $\sigma \subset X$ we fix a lift $\widetilde{\sigma}$. Consider the set of maps

$$
\bigcup_{\gamma \in \pi}\left\{\left.\gamma^{-1} g \gamma\right|_{\partial \widetilde{\sigma}}: \partial \widetilde{\sigma} \rightarrow Y^{(n-1)}\right\} \subset C\left(\partial \widetilde{\sigma}, Y^{(n-1)}\right) .
$$

By the construction of $g$, this set is finite. We fix an extension $\bar{g}_{\sigma^{\prime}}$ for each element of this set and define the extension $\bar{g}: X \rightarrow Y^{(n-1)}$ of $g$ by translations by $\pi$. We may assume that all maps $g_{i}$ and $\bar{g}_{\sigma^{\prime}}$ in the above construction are Lipschitz with the same Lipschitz constant since there are finitely many of them.

In the other direction, if there is a Lipschitz map $\bar{g}: \tilde{X} \rightarrow Y^{(n-1)}$ that coincides with $\tilde{f}$ on the $(n-2)$-dimensional skeleton, then the difference cochain $d_{\tilde{f}, \bar{g}}$ is almost equivariant. Indeed, for any $\lambda>0$ only finitely many homotopy classes in $\pi_{n-1}(Y)$ can be realized by $\lambda$-Lipschitz maps. Then the formula $\delta d_{\tilde{f}, \bar{g}}=C_{\bar{g}}-C_{\widetilde{f}}$ and the fact that $o \bar{g}=0$ imply that $o_{\tilde{f}}=0$.

Let $[e] \in \pi_{n-1}\left(\tilde{Y}^{(n-1)}\right)$ denote the element of the homotopy group defined by the attaching map of an $n$-cell $e$. Then the homomorphism $C_{\tilde{1}}: C_{n}(\tilde{Y}) \rightarrow \pi_{n-1}\left(\tilde{Y}^{(n-1)}\right)$ defined as $C_{\tilde{1}}(e)=[e]$ is an equivariant cocycle with the cohomology class $o_{\tilde{1}} \in$ $H_{a e}^{n}\left(\tilde{Y} ; \pi_{n-1}\left(Y^{(n-1)}\right)\right)$.

Proposition 3.6 (1) The cohomology class $o \tilde{f}$ from the above Proposition is the image under $\widetilde{f}^{*}$ of the class $o_{1} \in H_{a e}^{n}\left(\tilde{Y} ; \pi_{n-1}\left(Y^{(n-1)}\right)\right)$.

(2) The class $o_{1}$ comes under the homomorphism pert ${ }_{\pi}^{*}$ from the primary obstruction $\kappa_{1} \in H^{n}\left(Y ; \pi_{n-1}\left(Y^{(n-1)}\right)\right)$ to retract $Y$ to the $(n-1)$-dimensional skeleton.

Proof The first part is the naturality of obstructions for Lipschitz extension problems with respect to Lipschitz maps. Like in the case of classical obstruction theory, it follows from the definition.

The second part follows from definition (see Definition 3.4).

Proposition 3.7 Let $f: X \rightarrow B \pi$ be a Lipschitz map of a finite complex that induces an isomorphism of the fundamental groups and let $\tilde{f}: \tilde{X} \rightarrow E \pi$ be its lift to the universal coverings. Then for every $R>0$ the family of preimages of $R$-balls $\left\{\tilde{f}^{-1}\left(B_{R}(y)\right)\right\}_{y \in E \pi}$ is uniformly bounded where the metrics on $\widetilde{X}$ and $E \pi$ are induced from geodesic metrics on $X$ and $B \pi$.

Proof Since $\tilde{f}: \tilde{X} \rightarrow \tilde{f}(\tilde{X})$ is a quasi-isometry for the subset metric on $\tilde{f}(\tilde{X})$, the family $\left\{\tilde{f}^{-1}\left(B_{R}(y)\right)\right\}_{y \in \tilde{f}(\tilde{X})}$ is uniformly cobounded. Then, clearly, the family $\left\{\tilde{f}^{-1}\left(B_{R}(y)\right)\right\}_{y \in E \pi}$ is uniformly bounded. 
Theorem 3.8 Let $X$ be a finite $n$-complex with $\pi_{1}(X)=\pi$ and let $f: X \rightarrow B \pi$ be a Lipschitz map that induces an isomorphism of the fundamental groups. Then $\operatorname{dim}_{\mathrm{MC}} \tilde{X}<n, n \geq 3$, if and only if the above obstruction is trivial, $o_{\tilde{f}}=0$.

Proof If $\operatorname{dim}_{\mathrm{MC}} \tilde{X}<n$, then by Proposition 3.1 there is a Lipschitz cellular homotopy of $\tilde{f}: \tilde{X} \rightarrow E \pi$ to a map $g: \tilde{X} \rightarrow E \pi^{(n-1)}$. By Proposition 3.2, the map $g$ is almost equivariant. Then by Proposition 2.4, o $\tilde{f}=\tilde{f}^{*}\left(o_{1}\right)=g^{*} i^{*}\left(o_{1}\right)=0$ where $i: E \pi^{(n-1)} \subset E \pi$ is the inclusion.

We assume that $B \pi$ is a locally finite simplicial complex. If $o \tilde{f}=0$, then by Proposition 3.5 there is a $\lambda$-Lipschitz map $g: \tilde{X} \rightarrow E \pi^{(n-1)}$ for some $\lambda$ which agrees with $\tilde{f}$ on the $(n-2)$-skeleton $X^{(n-2)}$. We may assume that $f$ is also $\lambda-$ Lipschitz. Additionally, we assume that the diameter of each cell in $X$ is less than 1 . We show that the map $g$ is uniformly cobounded. In view of Proposition 3.7 it suffices to show that $g^{-1}(y) \subset \tilde{f}^{-1}\left(B_{3 \lambda}(y)\right)$ for all $y \in E \pi$. Let $x \in g^{-1}(y)$ and let $x \in e$ where $e$ is a cell in $\tilde{X}$. Since $X$ is $n$-dimensional, there is a point $v \in X^{(n-2)} \cap \operatorname{St}(\bar{e})$. Then $d_{\tilde{X}}(x, v)<2$ and $d(g(x), g(v))<2 \lambda$ where $d$ is the metric on $E \pi$ induced from a proper geodesic metric on $B \pi$. Thus, $d(y, \tilde{f}(v))<2 \lambda$. Since $\tilde{f}$ is $\lambda$-Lipschitz, by the triangle inequality and the fact that $\tilde{f}(v)=g(v)$,

$$
d(y, \tilde{f}(x))<d(y, g(v))+d(\tilde{f}(v), \tilde{f}(x))<3 \lambda,
$$

that is, $\tilde{f}(x) \in B_{3 \lambda}(y)$. Therefore, $x \in \tilde{f}^{-1}\left(B_{3 \lambda}(y)\right)$.

\section{Homology of groups}

Let $I$ denote the augmentation ideal of the group ring $\mathbb{Z} \pi$. We recall that the BersteinSchwarz class $\beta=\beta_{\pi} \in H^{1}(\pi ; I)$ is the first obstruction to the lift of $B \pi$ to $E \pi$ (see Švarc [12] and the paper [7] by the author and Rudyak). The following is called the Universality Theorem and it is stated without proof in [12]. A proof can be found in [7].

Theorem 4.1 For every $\pi$-module $L$ and every cohomology class $\alpha \in H^{k}(\pi ; L)$ there is a $\pi$-homomorphism $I^{k} \rightarrow L$ that takes $\beta^{k}$ to $\alpha$.

Here $\beta^{k}=\beta \smile \cdots \smile \beta$ is the $k$ times cup product and $I^{k}=I \otimes \cdots \otimes I$ is the $k$ times tensor product over $\mathbb{Z}$. We recall that the cup product $x \smile y$ of classes $x \in H^{*}(X ; A)$ and $y \in H^{*}(X ; B)$ is defined for any modules $A$ and $B$ with $x \smile y \in H^{*}(X ; A \otimes B)$ (see Brown [4]). 
Let $f: M \rightarrow B \pi$ be a map that induces an isomorphism of the fundamental groups. The image $f^{*}\left(\beta_{\pi}\right) \in H^{1}(M ; I)$ of the Berstein-Schwarz class of $\pi$ is denoted by $\beta_{M}$ and is called Berstein-Schwarz class of $M$.

We will use the notations $H_{a e}^{*}(\pi ; L), H_{*}^{l f, a e}(\pi ; L), \operatorname{pert}_{\pi}^{*}$ and pert ${ }_{*}^{\pi}$ for $H_{a e}^{*}(E \pi ; L)$, $H_{*}^{l f, a e}(E \pi ; L), \operatorname{pert}_{B \pi}^{*}$ and $\operatorname{pert}_{*}^{B \pi}$ respectively. Also we will use the notation $H_{*}(\pi)$ for $H_{*}(\pi ; \mathbb{Z})$.

Theorem 4.2 For a closed oriented $n$-manifold $M$ the following are equivalent:

(1) $\operatorname{dim}_{\mathrm{MC}} \widetilde{M}<n$;

(2) $f_{*}([M]) \in \operatorname{ker}\left(\operatorname{pert}_{*}^{\pi}\right)$ where $f: M \rightarrow B \pi$ is the map classifying the universal covering $\widetilde{M}$ of $M$.

(3) $\left(\beta_{M}\right)^{n} \in \operatorname{ker}\left(\operatorname{pert}_{M}^{*}\right)$.

Proof $(1) \Rightarrow(2)$ Let $f: M \rightarrow B \pi$ be a cellular Lipschitz map classifying the universal cover $\widetilde{M}$ of $M$ and let $\widetilde{f}: \widetilde{M} \rightarrow E \pi$ be a lift. If $\operatorname{dim}_{\mathrm{MC}} \widetilde{M}<n$, then by Proposition 3.1 there is a Lipschitz cellular homotopy of $\bar{f}: \tilde{X} \rightarrow E \pi$ to a map $g: \tilde{X} \rightarrow E \pi^{(n-1)}$. By Proposition 3.2, it is almost equivariant. Then by Proposition 2.4 it follows that $\tilde{f}_{*}\left(\operatorname{pert}_{*}^{M}([M])\right)=0$. Therefore, $\operatorname{pert}_{*}^{\pi}\left(f_{*}([M])\right)=0$ and hence, $f_{*}([M]) \in \operatorname{ker}\left(\operatorname{pert}_{*}^{\pi}\right)$.

(2) $\Rightarrow$ (3) If $f_{*}([M]) \in \operatorname{ker}\left(\operatorname{pert}_{*}^{\pi}\right)$, then $\operatorname{pert}_{*}^{\pi}\left(f_{*}([M]) \cap \beta^{n}\right)=0$. Since we may assume that the restriction of $f$ to the 1-skeleton of $M$ is a homeomorphism of 1-skeletons, the commutative diagram

$$
\begin{array}{ccc}
H_{0}^{l f, a e}\left(\widetilde{M} ; I^{n}\right) & \stackrel{\bar{f}_{*}}{\longrightarrow} & H_{0}^{l f, a e}\left(E \pi ; I^{n}\right) \\
\operatorname{pert}_{*}^{M \uparrow} & & \operatorname{pert}_{*}^{\pi} \uparrow \\
H_{0}\left(M ; I^{n}\right) & \stackrel{f_{*}}{\longrightarrow} & H_{0}\left(B \pi ; I^{n}\right)
\end{array}
$$

has isomorphisms for horizontal arrows. Therefore, $\operatorname{pert}_{*}^{M}\left([M] \cap\left(f^{*} \beta\right)^{n}\right)=0$. Thus, $\operatorname{pert}_{*}^{M}([M]) \cap \operatorname{pert}_{M}^{*}\left(\left(f^{*} \beta\right)^{n}\right)=0$. By the Poincare Duality, pert ${ }_{M}^{*}\left(\left(\beta_{M}\right)^{n}\right)=0$.

(3) $\Rightarrow$ (1) By Proposition 3.6, $o_{\tilde{f}}=\operatorname{pert}_{M}^{*}\left(f^{*}\left(\kappa_{1}\right)\right)$. By the Universality Theorem there is a coefficient homomorphism $\psi: I^{n} \rightarrow \pi_{n-1}\left(B \pi^{(n-1)}\right)=L$ such that the induced homomorphism of the $n$th cohomology groups takes $\beta^{n}$ to $\kappa_{1}$. Therefore, $\psi$ induces the commutative diagram

$$
\begin{array}{ccc}
H_{a e}^{n}\left(\widetilde{M} ; I^{n}\right) & \stackrel{\psi_{*}}{\longrightarrow} & H_{a e}^{n}(\widetilde{M} ; L) \\
\operatorname{pert} \uparrow & \\
H^{n}\left(M ; I^{n}\right) \stackrel{\psi_{*}}{\longrightarrow} & H^{n}(M ; L)
\end{array}
$$


where

$$
\left.o_{\tilde{f}}=\operatorname{pert}_{M}^{*}\left(f^{*}\left(\kappa_{1}\right)\right)=\operatorname{pert}_{M}^{*}\left(\psi_{*}\left(\beta_{M}\right)^{n}\right)\right)=\psi_{*}\left(\operatorname{pert}_{M}^{*}\left(\left(\beta_{M}\right)^{n}\right)\right)=0 .
$$

Then by Theorem $3.8 \operatorname{dim}_{\mathrm{MC}} \widetilde{M}<n$.

We note that the subset of $n$-homology classes of $H_{n}(\pi)$ which can be realized by an $n$-manifolds forms a subgroup. We denote this subgroup by $R H_{n}(\pi)$ and call it the representable $n$-homology group. Using the surgery one can show that for $n \geq 4$ a realization $f: M \rightarrow B \pi$ of a given class from $R H_{n}(\pi)$ can be taken such that $f$ induces an isomorphism of the fundamental groups.

Definition 4.3 We define the group of small macroscopic dimension homology classes as $H_{n}^{\mathrm{sm}}(\pi)=\operatorname{ker}\left(\operatorname{pert}_{*}^{\pi}\right) \cap R H_{n}(\pi) \subset H_{n}(\pi)$.

Corollary 4.4 For a closed orientable $n$-manifold $M, \operatorname{dim}_{\mathrm{MC}} \widetilde{M}<n$ if and only if $f_{*}([M]) \in H_{n}^{\mathrm{sm}}(\pi)$.

We note that this Corollary is a statement in spirit of Brunnbauer and Hanke [5]. For several classes of large manifolds they proved similar statements. For example, they proved the following

Theorem 4.5 For every finitely presented group $\pi$ and every $n$ there is a subgroup $H_{n}^{\mathrm{sm}}(\pi ; \mathbb{Q}) \subset H_{n}(\pi ; \mathbb{Q})$ such that a closed orientable $n$-manifold $M$ has the hypereuclidean universal cover $\widetilde{M}$ if and only if $f_{*}([M]) \in H_{n}^{\mathrm{sm}}(\pi ; \mathbb{Q})$.

We recall that an $n$-manifold $N$ is called hyper-euclidean if there is a Lipschitz map $p: N \rightarrow \mathbb{R}^{n}$ of non-zero degree. Theorem 4.5 and all other results in [5] are rational. It is unclear if our Corollary can be stated in terms of the group homology with rational coefficients.

We conclude this section with the following observation about homologies of a group.

Theorem 4.6 For every group $\pi$ and any $n>0$,

$$
H_{n}(\pi)=H_{n-1}(\pi ; I)=H_{n-2}\left(\pi ; I^{2}\right)=\cdots=H_{1}\left(\pi ; I^{n-1}\right)
$$

and

$$
H_{n}(\pi)=\operatorname{ker}\left\{H_{0}\left(\pi ; I^{n}\right) \rightarrow H_{0}\left(\pi ; I^{n-1} \otimes \mathbb{Z} \pi\right)\right\}
$$

or to state the same differently,

$$
H_{n}(\pi)=\operatorname{ker}\left\{I^{n} \otimes_{\pi} \mathbb{Z} \stackrel{(1 \otimes i) \otimes 1}{\longrightarrow}\left(I^{n-1} \otimes \mathbb{Z} \pi\right) \otimes_{\pi} \mathbb{Z}\right\}
$$

where $i: I \rightarrow \mathbb{Z} \pi$ is the embedding. 
Proof The first chain of equalities follow from the homology long exact sequence defined by the short exact sequence of coefficients

$$
0 \rightarrow I^{k} \rightarrow I^{k-1} \otimes \mathbb{Z} \pi \rightarrow I^{k-1} \rightarrow 0
$$

and the fact that $H_{i}\left(B \pi ; I^{k-1} \otimes \mathbb{Z} \pi\right)=0$ for $i>0$. The latter is due to the facts that the reduced homologies of a group with coefficients in a projective module are trivial (see Brown [4]) and the modules $I^{k-1} \otimes \mathbb{Z} \pi$ are projective (see the paper [7] by the author and Rudyak). Here we use the convention $I^{0}=\mathbb{Z}$.

The second equality follows from the facts that $H_{n}(\pi)=H_{1}\left(\pi ; I^{n-1}\right)$ and the remainder of the coefficients exact sequence is

$$
0 \rightarrow H_{1}\left(\pi ; I^{n-1}\right) \rightarrow H_{0}\left(\pi ; I^{n}\right) \rightarrow H_{0}\left(\pi ; I^{n-1} \otimes \mathbb{Z} \pi\right) \rightarrow H_{0}\left(\pi ; I^{n-1}\right) .
$$

The last equality follows from definition of 0 -dimensional homology:

$$
H_{0}(\pi ; L)=\operatorname{coinv}(L)=L \otimes_{\pi} \mathbb{Z}
$$

for every $\pi$-module $L$.

\section{Main Result}

The following theorem is due to Block and Weinberger [2].

Theorem 5.1 For a finite complex $K$ with the universal covering $\tilde{K}$ and the fundamental group $\pi$ and for the trivial $\pi$-module $\mathbb{Z}$, the equality $H_{0}^{l f, a e}(\tilde{K} ; \mathbb{Z})=0$ holds true if and only if $\pi$ is not amenable.

Here is our main result.

Theorem 5.2 For all $n \geq 4$ there is a closed rationally essential $n$-manifold $M$ with the fundamental group $\pi_{1}(M)=\mathbb{Z}^{n} \times F_{2}$ such that $\operatorname{dim}_{\mathrm{MC}} \widetilde{M}<n$.

Proof We note that $B \pi=T^{n} \times\left(S^{1} \vee S^{1}\right)$ for $\pi=\mathbb{Z} \times F_{2}$ where $T^{n}$ is the $n$-torus. Consider the natural inclusion of $T^{n}$ into $B \pi$. Then the image of the fundamental class $\left[T^{n}\right]$ in $H_{n}(B \pi)$ is $\left[T^{n}\right] \otimes 1$ where $1 \in H_{0}\left(S^{1} \vee S^{1}\right)$. By Theorem 5.1, $\operatorname{pert}_{*}^{F_{2}}(1)=0$. Therefore, in view of Proposition 2.6,

$$
\operatorname{pert}_{*}^{\pi}\left(\left[T^{n}\right] \otimes 1\right)=\operatorname{pert}_{*}^{\mathbb{Z}^{n}}\left(\left[T^{n}\right]\right) \otimes \operatorname{pert}_{*}^{F_{2}}(1)=0 .
$$

By a surgery in dimension 0 and 1 performed on the torus $T^{n}$ we can obtain a manifold $M$ together with a map $f: M \rightarrow B \pi$ inducing isomorphism of the fundamental groups and such that $f([M])=\left[T^{n}\right] \otimes 1$. 
Namely, we consider a map $q: T^{n} \rightarrow T^{n}$ that collapses a closed $n$-disc $D \subset T^{n}$ to the base point $x_{0} \in T^{n}$. Let $\alpha_{1}$ and $\alpha_{2}$ be generators of $F_{2}$. For $i=1,2$ we do the surgery on $T^{n}$ in dimension 0 by attaching a solid 1 -handle $H_{i} \cong B^{n} \times[0,1]$ to $D$ along $B^{n} \times\{0,1\}$ preserving orientation. We do it in a way that the bases of the handles $H_{1}$ and $H_{2}$ do not intersect. Let $W=T^{n} \cup H_{1} \cup H_{2}, \stackrel{\circ}{H}_{i}=\operatorname{Int} B^{n} \times[0,1]$, and $N=W \backslash\left(\stackrel{\circ}{H}_{1} \cup \stackrel{\circ}{H}_{2}\right)$. Note that $N$ is an orientable $n$-manifold. Clearly, the fundamental class $[N]$ is homologous to $\left[T^{n}\right]$ in $W$. For $i=1,2$ we define a map $f_{i}: H_{i} \rightarrow B \pi$ by collapsing $H_{i}$ to the interval $[0,1]$ and then by sending this interval along a loop in $\left\{x_{0}\right\} \times\left(S^{1} \vee S^{1}\right) \subset B \pi$ representing $\alpha_{i}$. Note that the union $f_{1} \cup f_{2} \cup q$ defines a continuous map $\bar{q}: W \rightarrow B \pi$. Then the restriction $f^{\prime}=\left.\bar{q}\right|_{N}: N \rightarrow B \pi$ induces an epimorphism of the fundamental groups and takes the fundamental class $[N]$ to $q_{*}\left(\left[T^{n}\right]\right)=\left[T^{n}\right] \otimes 1$.

Next, we do a surgery on $N$ in dimension 1 to kill the kernel of the homomorphism $f_{*}^{\prime}: \pi_{1}(N) \rightarrow \pi_{1}(B \pi)=\pi$. It is known that the kernel of any epimorphism of finitely presented groups is normally generated by finitely many elements. In our case, since $f_{*}^{\prime}$ is the abelianization, these elements can be taken to be the commutators of generators of $\pi_{1}(N)$. Let $\gamma_{1}, \ldots, \gamma_{m}$ be such elements. For every $j=1, \ldots, m$ we take a loop in $N$ representing $\gamma_{i}$ and approximate it by a smoothly embedded simple curve $C_{j}$. In view of the orientability of $N$, the normal bundle to $C_{j}$ is trivial and we can perform the killing surgery. That surgery can be viewed as attaching to $N$ a solid 2-handle $H_{j}^{2}=B^{n-1} \times B^{2}$ along the tubular neighborhood of $C_{j}$ and then removing Int $B^{n-1} \times B^{2}$. Our manifold $M$ is the result of this (iterated) surgery. Let $W^{2}=N \cup \cup_{j} H_{j}^{2}$. Note that $[N]$ is homologous to $[M]$ in $W^{2}$. Since $\gamma_{j}$ is in the kernel of $f_{*}^{\prime}$, the map $f^{\prime}$ is extendable to $H_{j}^{2}$. Let $\bar{f}: W^{2} \rightarrow B \pi$ be an extension. Note that $\bar{f}$ induces an isomorphism of the fundamental groups. Since $\operatorname{dim} H_{j}^{2} \geq 5$, by the general position property any 2-dimensional disc in $W^{2}$ can be removed from the core $b_{0} \times B^{2}$ where $b_{0} \in B^{n-1}$ is the center. In view of the deformation retractions of $H_{j}^{2} \backslash\left(b_{0} \times B^{2}\right)$ onto $\partial B^{n-1} \times B^{2}$, the inclusion $M \subset W^{2}$ induces an isomorphism of the fundamental groups. Then $f=\left.\bar{f}\right|_{M}$ induces an isomorphism of the fundamental groups and takes the fundamental class $[M]$ to $f_{*}^{\prime}([N])=\left[T^{n}\right] \otimes 1$.

Since $\operatorname{pert}_{*}^{\pi}\left(\left[T^{n}\right] \otimes 1\right)=0$, by Theorem $3.8, \operatorname{dim}_{\mathrm{MC}} \widetilde{M}<n$.

Remark The free group $F_{2}$ in the Theorem 5.2 can be replaced by any non-amenable finitely presented group and the torus $T^{n}$ can be replaced by any rationally essential $n$-manifold $N$. 


\section{References}

[1] G Bell, A Dranishnikov, On asymptotic dimension of groups acting on trees, Geom. Dedicata 103 (2004) 89-101 MR2034954

[2] J Block, S Weinberger, Aperiodic tilings, positive scalar curvature and amenability of spaces, J. Amer. Math. Soc. 5 (1992) 907-918 MR1145337

[3] D Bolotov, A Dranishnikov, On Gromov's scalar curvature conjecture, Proc. Amer. Math. Soc. 138 (2010) 1517-1524 MR2578547

[4] KS Brown, Cohomology of groups, Graduate Texts in Mathematics 87, Springer, New York (1994) MR1324339 Corrected reprint of the 1982 original

[5] M Brunnbauer, B Hanke, Large and small group homology, J. Topol. 3 (2010) 463486 MR2651368

[6] A Dranishnikov, Macroscopic dimension and essential manifolds, from: "Proceedings of the Conference dedicated to the 75th anniversary of the Steklov Mathematical Institute, Moscow, 2009”, Proc. Steklov Inst. Math.

[7] A N Dranishnikov, Y B Rudyak, On the Berstein-Svarc theorem in dimension 2, Math. Proc. Cambridge Philos. Soc. 146 (2009) 407-413 MR2475974

[8] S M Gersten, Cohomological lower bounds for isoperimetric functions on groups, Topology 37 (1998) 1031-1072 MR1650363

[9] M Gromov, Filling Riemannian manifolds, J. Differential Geom. 18 (1983) 1-147 MR697984

[10] M Gromov, Asymptotic invariants of infinite groups, from: "Geometric group theory, Vol. 2 (Sussex, 1991)”, London Math. Soc. Lecture Note Ser. 182, Cambridge Univ. Press, Cambridge (1993) 1-295 MR1253544

[11] M Gromov, Positive curvature, macroscopic dimension, spectral gaps and higher signatures, from: "Functional analysis on the eve of the 21st century, Vol. II (New Brunswick, NJ, 1993)", Progr. Math. 132, Birkhäuser, Boston (1996) 1-213 MR1389019

[12] A S Švarc, The genus of a fibered space, Trudy Moskov. Mat. Obšč. 10 (1961) 217-272 MR0154284

Department of Mathematics, University of Florida

358 Little Hall, Gainesville, Florida 32611-8105, USA

dranish@math.ufl.edu

Proposed: Steve Ferry

Received: 29 April 2010

Seconded: David Gabai, Ronald J Stern

Revised: 11 April 2011 